\title{
Successful laparoscopic extirpation of a vasoactive intestinal polypeptide-secreting neuroblastic tumor originating from the right adrenal gland: A report of an infantile case
}

\author{
Koshiro Sugita ${ }^{1}$, Tatsuru Kaji ${ }^{1}$, Ayaka Nagano ${ }^{1}$, Mitsuru Muto ${ }^{1}$, Takuro Nishikawa ${ }^{1}$, \\ Hiromu Masuda ${ }^{1}$, Ryo Imakiire ${ }^{1}$, Yasuhiro Okamoto $^{1}$, Mari Imamura ${ }^{2}$, and Satoshi Ieiri ${ }^{1}$ \\ ${ }^{1}$ Research Field in Medical and Health Sciences, Medical and Dental Area, Research and \\ Education Assembly, Kagoshima University \\ ${ }^{2}$ Kagoshima Prefecture Oshima Hospital
}

September 11, 2020

\begin{abstract}
We herein report a 10-month-old female infant with a 4-month history of diarrhea with electrolyte abnormalities and growth impairment. A 4-cm right adrenal tumor was detected by computed tomography. No metastasis or accumulation on I123metaiodobenzylguanidine scintigraphy was recognized in the tumor. A vasoactive intestinal peptide-secreting neuroblastic tumor was suspected, and octreotide was started, but the diarrhea persisted. Tumor extirpation was performed laparoscopically. After tumor removal, the symptoms improved immediately, and her growth caught up by nine months after surgery. A minimally invasive approach for pediatric solid tumor is difficult, especially for neuroblastoma, but may be beneficial for the patient's recovery.
\end{abstract}

\section{INTRODUCTION}

Diarrhea is a frequent visceral symptom of pediatric patients. Diarrhea that does not improve after more than four weeks is defined as chronic diarrhea [1]. Persistent chronic diarrhea can induce electrolyte abnormalities and growth impairment. Vasoactive intestinal polypeptide (VIP)-secreting tumor (VIPoma) of neuroendocrine tumor can cause chronic diarrhea, which may manifest as Verner-Morrison syndrome (hypokalemia, acidosis, and severe dehydration) [2] or WDHA syndrome (watery diarrhea, hypokalemia, achlorhydria, and acidosis) [3].

VIPoma can be diagnosed by measuring the serum VIP concentration. However, the measurement of serum VIP is not currently available on a commercial basis in Japan. Therefore, imaging modalities and pathological examinations are important and essential for a definitive diagnosis.

Several recent reports have described laparoscopic surgery for neuroblastoma [4-6], but no literature regarding laparoscopic surgery for VIP-secreting neuroblastic tumor (VIPNT) in pediatric patients has been found.

We herein report our case of very-low-risk VIPNT in an infant who underwent successful laparoscopic surgery.

\section{CASE DESCRIPTION}

\subsection{Patient}

The patient was a 10-month-old female. She had been treated for gastrointestinal allergy from six months old. Watery diarrhea persisted for 4 months, and growth impairment (body height $65 \mathrm{~cm} ;-2.96$ standard deviations [SD], body weight $6.1 \mathrm{~kg} ;-2.8 \mathrm{SD}$ ), dehydration, and hypokalemia were recognized. A 4-cm right 
adrenal tumor was detected on computed tomography, but lymphocytic and distant metastasis were not detected (Fig. 1A, B). ${ }^{123}$-metaiodobenzylguanidine scintigraphy (Fig. 1C) showed no isotope accumulation in the tumor. Laboratory data showed elevated levels of neuron-specificenolase $(42.2 \mathrm{ng} / \mathrm{mL})$, urinary vanillylmandelic acid $(24.8 \mu \mathrm{g} / \mathrm{mg}$ Creatinine), and urinary homovanillic acid (51.2 $\mu \mathrm{g} / \mathrm{mg}$ Creatinine). Dopamine $(6023 \mathrm{pg} / \mathrm{mL})$ was elevated, but adrenaline $(9.0 \mathrm{pg} / \mathrm{mL})$ and noradrenaline $(82.0 \mathrm{pg} / \mathrm{mL})$ were within normal levels. Based on the clinical course, imaging findings, and laboratory data, a VIPNT of adrenal was clinically diagnosed.

Octreotide therapy was started, but the diarrhea persisted. The clinical stage was defined as L1 according to the International Neuroblastoma Risk Group (INRG) staging system. In addition, image-defined risk factors (IDRF) were negative, with no tumor infiltration into the surrounding organs or encasing the primary vessels. Tumor extirpation by laparoscopic surgery was planned based on the staging, IDRF, and tumor size.

\subsection{Surgical procedure}

Laparoscopic surgery was performed by 4 inserted ports along with the open Hasson procedure, as follows: 12 -mm port for the scope; umbilical position, 5 -mm port; sub xiphoid for the operator's right hand, right flank region for the operator's left hand and right upper abdomen for the assistant (Fig. 2A). To obtain adequate visibility of the inferior space of the liver, the round ligament of the liver was retracted with 20 silk using a 19-G percutaneous loop needle insertion device. In addition, the snake liver retractor was inserted through the trocar of the right upper abdomen to elevate the right lobe of the liver [7]. The tumor, located at the right side of the duodenum, was carefully dissected in a sharp and blunt manner and never directly grasped with forceps to prevent rupture and spillage of the tumor (Fig. 2B). The feeding artery and drainage vein were coagulated and divided using the vessel sealing system (Fig. 2C). The tumor was completely excised without rupture or spillage. The umbilical wound for the optical port was extended, and the tumor was extracted using an endsurgical bag. The operative time was 149 minutes, with 2-ml blood loss.

\subsection{Pathological findings of the extirpated specimen}

Hematoxylin-eosin staining revealed $<50 \%$ stroma of Schwann cells and a low mitosis karyorrhexis-index (MKI) (Fig. 2D), and immunohistochemical staining showed positivity for VIP (Fig. 2E). The definitive pathological diagnosis according to the international neuroblastoma pathology classification of the Shimada system [8] was neuroblastoma, differentiating subtype with a low MKI, favorable histology, MYCN not amplified.

\subsection{Postoperative course}

The postoperative course was uneventful, and the patient started oral intake two days after surgery. Her diarrhea immediately improved after surgery. According to the INRG risk classification, the patient was classified into the very-low-risk group, and postoperative chemotherapy was unnecessary. The patient's growth caught up at 9 months after surgery (body height $77 \mathrm{~cm},-1.2 \mathrm{SD}$; body weight $9.62 \mathrm{~kg},-0.54 \mathrm{SD}$ ) and she has had an event-free survival.

\section{DISCUSSION}

VIPoma in adults is primarily pancreatic tumor, with the tail of the pancreas being the most common site of origin [9]. The majority of VIPomas in pediatric patients are ganglioneuroblastomas or ganglioneuromas originating in the adrenal medulla or sympathetic neural crest, in contrast to adult cases [10]. In our institution, VIPoma has been found in only 3 patients (2.7\%) among 111 with neuroblastic tumors. Among our patients with neuroblastic tumors, minimal invasive surgery (MIS) was performed in about $12.0 \%$ of cases, so MIS for VIPNT seems to be an extremely rare application.

VIPNT is divided into cases preceded by diarrhea (primary type) and cases becoming clinically evident after chemotherapy [11]. Whether neoadjuvant chemotherapy or surgery should be prioritized for patients with VIPNT and IDRF positivity or metastases has been controversial. In such cases, tumor resection after a 
biopsy to confirm the tumor biology and neoadjuvant chemotherapy is recommended. However, as in our case, the effects of somatostatin analogs on VIPNT may be limited [12,13]. Kabalan et al. reported the efficacy of debulking and steroids for symptoms in cases of primary type with unresectable VIPNT [13]. Their case was preceded by two courses of neoadjuvant chemotherapy, but the diarrhea symptoms did not improve. Control of VIPNT symptoms may be difficult with chemotherapy or medical treatment [11]. A surgical approach was the first-line treatment in our case to control diarrhea. While extirpation of the VIPNT should be the first choice, volume reduction might be a suitable second choice in unresectable cases with positive IDRF or amplified MYCN following chemotherapy.

MIS for VIPoma has mainly been reported in cases of pancreatic neoplasms in adult patients [14]. Pediatric patients with solid tumors may also enjoy certain benefits of MIS in terms of a faster recovery, less pain, better cosmetic results, and earlier start of adjuvant treatment [5]. The primary type of VIPNT has a favorable prognosis of $90 \%$ [11] and may be suitable for MIS among pediatric patients. The International Pediatric Endosurgery Group (IPEG) issued guidelines in 2010 for laparoscopic adrenalectomy based on level III evidence and confirmed its feasibility without absolute contraindications [15].

The most important factor to consider for MIS is the tumor size. The IPEG-issued guidelines recommend a maximum size of $6 \mathrm{~cm}$ in their criteria [15]. Our case had a maximum diameter of approximately $4 \mathrm{~cm}$, so complete tumor excision by laparoscopic surgery was considered feasible. In addition, the preoperative evaluation of IDRF is also important for performing laparoscopic surgery [16]. The risk of open conversion should be considered preoperatively, as radiological findings may not be compatible with the intraoperative findings [4]. Our case was a feasible indication for laparoscopic surgery with a tumor size of $4 \mathrm{~cm}$ and negative IDRF.

\section{Conclusions}

In this case, tumor extirpation was more effective for symptom control of VIPoma than medication. MIS is a favorable surgical treatment option for pediatric VIPoma and should be considered for treating neuroblastic tumors.

\section{Compliance with ethical standards}

The authors declare no conflicts of interest in association with the present study.

\section{Acknowledgments}

We thank Brian Quinn for his comments and help with the article. This study was supported by Grantsin-Aid for Scientific Research from the Japan Society for the Promotion of Science (JSPS, Nos. 16K11350, 19K09150), research grant from JFE (The Japanese Foundation for Research and Promotion of Endoscopy), research grant from J-CASE (Japanese Consortium of Advanced Surgical Endoscopy), research grant from Kyushu Society of Endoscopic and Robotic Surgery, research grant of Karl Storz Award from Japan Society for Endoscopic Surgery, research grant from The Mother and Child Health Foundation, research grant from the Kawano Masanori Memorial Public Interest Incorporated Foundation for Promotion of Pediatrics, Research Grant from Princess Takamatsu Cancer Research Fund.

\section{References}

1. Benninga MA, Faure C, Hyman PE et al. Childhood Functional Gastrointestinal Disorders: Neonate/Toddler. Gastroenterology2016 Feb 15; DOI: 10.1053/j.gastro.2016.02.016.

2. Verner JV, Morrison AB. Islet cell tumor and a syndrome of refractory watery diarrhea and hypokalemia. Am J Med 1958; 25: 374-380

3. Bloom SR, Polak JM, Pearse AG. Vasoactive intestinal peptide and watery-diarrhoea syndrome. Lancet 1973; 2 : $14-16$

4. Fuchs J, Schafbuch L, Ebinger M et al. Minimally invasive surgery for pediatric tumors - current state of the art. Front Pediatr 2014; 2: 48 
5. Malkan AD, Loh AH, Sandoval JA. Minimally invasive surgery in the management of abdominal tumors in children. J Pediatr Surg 2014; 49: 1171-1176

6. Boo YJ, Goedecke J, Muensterer OJ. Pediatric oncologic endosurgery.Int J Surg Oncol (N Y) 2017 Aug 2: e30. DOI:10.1097/IJ9.0000000000000030.2017.08.20

7. Harumatsu T, Nagai T, Yano K et al. Differential advantage of liver retraction methods in laparoscopic fundoplication for neurologically impaired patients: a comparison of three kinds of procedures.Pediatr Surg Int 2020; 36: 591-596

8. Shimada H, Ambros IM, Dehner LP et al. The International Neuroblastoma Pathology Classification (the Shimada system).Cancer 1999; 86: 364-372

9. Anderson CW, Bennett JJ. Clinical Presentation and Diagnosis of Pancreatic Neuroendocrine Tumors. Surg Oncol Clin N Am 2016; 25: 363-374

10. Belei OA, Heredea ER, Boeriu E et al. Verner-Morrison syndrome. Literature review. Rom J Morphol Embryol 2017; 58: 371-376

11. Bourdeaut F, de Carli E, Timsit S et al. VIP hypersecretion as primary or secondary syndrome in neuroblastoma: A retrospective study by the Société Française des Cancers de l'Enfant (SFCE). Pediatr Blood Cancer 2009; 52: 585-590

12. Tiedemann K, Pritchard J, Long R et al. Intractable diarrhoea in a patient with vasoactive intestinal peptide-secreting neuroblastoma. Attempted control by somatostatin. Eur J Pediatr 1981; 137: 217-219

13. Kabalan P, Gifford AJ, Ziegler DS. Unresectable VIP-secreting neuroblastoma: Efficacy of debulking and steroids for symptom control.Pediatr Blood Cancer 2018; 65: e27358

14. Fernández-Cruz L, Cosa R, Blanco L et al. Curative laparoscopic resection for pancreatic neoplasms: a critical analysis from a single institution. J Gastrointest Surg 2007; 11: 1607-1621; discussion 1621-1602

15. International Pediatric Endosurgery Group guidelines for the surgical treatment of adrenal masses in children. J Laparoendosc Adv Surg Tech A 2010; 20: vii-ix

16. Parhar D, Joharifard S, Lo AC et al. How well do image-defined risk factors (IDRFs) predict surgical outcomes and survival in patients with neuroblastoma? A systematic review and meta-analysis. Pediatr Surg Int 2020; 36: 897-907

\section{Legends for Figures}

Figure 1. The radiographical assessment of enhanced computed tomography and $\mathrm{I}^{123}$ metaiodobenzylguanidine scintigraphy findings.

Abdominal enhanced computed tomography showed that the right adrenal tumor surrounded by a yellow circle had grown to $4 \mathrm{~cm}$ in diameter (A: transverse plane, B: coronal plane). $\mathrm{I}^{123}$-metaiodobenzylguanidine scintigraphy showed no uptake (C: transverse plane).

Figure 2. Intraoperative findings, including the port layout for laparoscopic surgery andhistopathological findings.

The port layout at the operation showed a 12-mm port, mainly used as a camera port, and a 5 -mm port, mainly used as a working port (A). It was important to keep the tumor as intact as possible, so we grasped the tissue surrounding the tumor (B). Blood vessels were cut using the vessel sealing system (C). Hematoxylineosin stain showed that the surgical specimen consisted of round cells with rich neurofibrils and $<50 \%$ stroma of Schwann cells. The tumor cells had round nuclei and eosinophilic vesicles and large cells that prominently showed differentiation into ganglion cells (D). Immunohistochemical staining showed positive findings for VIP (E). 
Figure 1. The radiographical assessment of enhanced computed tomography and $\mathrm{I}^{123}$ metaiodobenzylguanidine scintigraphy findings.

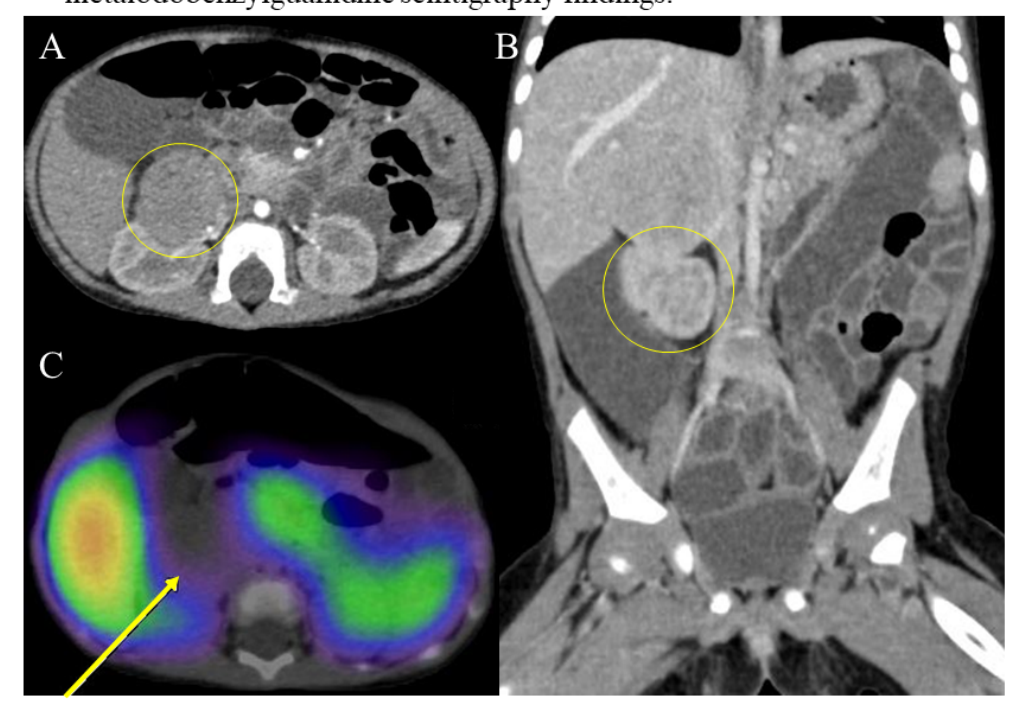

Figure 2. Intraoperative findings, including the port layout for laparoscopic surgery and histopathological findings.
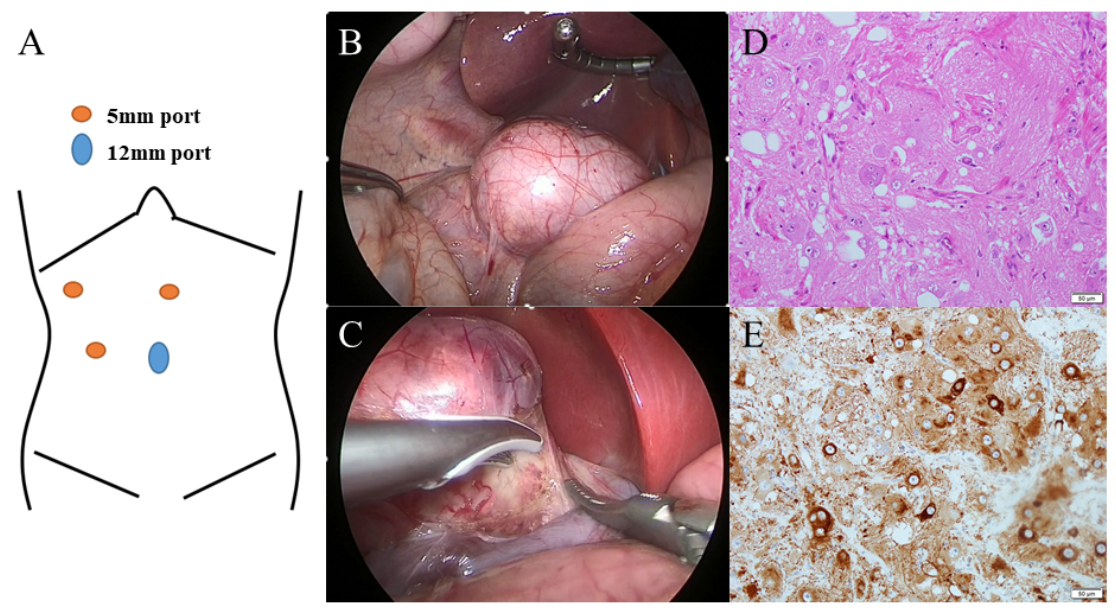